\title{
Mycobacterium avium subspecies paratuberculosis and goblet cells: are Barrett's esophagus and esophageal adenocarcinoma zoonotic infectious diseases?
}

Ellen S. Pierce*

13212 East Blossey Avenue, Spokane Valley, Washington, USA

Article Info

\section{Article Notes}

Received: September 11, 2018

Accepted: October 25, 2018

\section{*Correspondence:}

Ellen S. Pierce, M.D., 13212 East Blossey Avenue, Spokane Valley, Washington, USA; Telephone No: +1 509922 4562; Fax No: +1 509842 8522; Email: ellenpiercemd@gmail.com

C 2018 Pierce ES. This article is distributed under the terms of the Creative Commons Attribution 4.0 International License.

\section{Keywords}

Esophageal adenocarcinoma

Barrett's esophagus

Intestinal metaplasia

Goblet

Paratuberculosis

Infectious etiology
There has been a great increase in the incidence of esophageal adenocarcinoma (EAC) in western so-called developed countries in the past several decades ${ }^{1-3}$. This marked increase has led some to speculate that "changing environmental factors and microbial agents"4 may be contributing factors.

A possible infectious cause of EAC and associated Barrett's esophagus (BE) is Mycobacterium avium subspecies paratuberculosis (MAP). MAP, a long suspected cause of Crohn's disease, is a probable zoonosis ${ }^{5}$, a microorganism that causes disease in humans and is transmitted to humans from animals.

MAP is present in an infected domestic ruminant's manure, milk, and muscle tissue. Humans can become infected with MAP by swallowing MAP organisms in MAP-contaminated potable water, MAP-contaminated milk and other dairy products, and MAP-contaminated meat, or inhaling and then swallowing MAPcontaminated water aerosolized from rivers and lakes.

Presumably MAP-associated Crohn's disease is known to cause oral $^{6}$, esophageal ${ }^{7}$, and gastric lesions ${ }^{8}$. MAP organisms are present in oral biopsies from patients with Crohn's disease ${ }^{9}$.

Goblet cell proliferation that occurs in locations where goblet cells already exist is called goblet cell hyperplasia. Goblet cell hyperplasia of the small and large intestines is a known result of infection with enteropathogenic bacteria and parasites ${ }^{10}$. MAP has the little-known ability to cause acute ${ }^{11}$ and chronic ${ }^{12}$ goblet cell hyperplasia in the small and large intestines of its natural hosts, domestic ruminants.

MAP has a predilection for human intestinal goblet cells. A single striking study shows MAP organisms actively migrating within the mucus of human fetal small intestines to a position directly above goblet cells, gathering in dense aggregations, and then heavily invading the goblet cells ${ }^{13}$. MAP also appears perfectly capable of invading not just goblet cells, but every cell type in the intestines. A single study documents that MAP can directly infect all cell types of bovine intestinal epithelium, but causes the proliferation or hyperplasia only of infected goblet cells ${ }^{11}$.

As MAP organisms actively migrate within human fetal small intestinal mucus to the apical surface of goblet cells and then actively invade the apical granule portion of those goblet cells, MAP organisms may be able to migrate within the mucus layer of the stomach to the apical surface of cardiac surface mucus cells and then 
actively invade those cardiac surface mucus cells. If MAP preferentially colonizes the mucus above or the mucus within cardiac surface mucus cells, then the pathologic changes MAP causes will begin in the gastric cardia and work their way up. MAP infected cardiac surface mucus cells that proliferate and 'spill' upward would explain why $\mathrm{BE}$ begins at the esophagogastric junction and spreads proximally, referred to as "proximal migration"14.

MAP infection of the gastric cardia mucus or surface mucus cells, causing their proximal migration, explains what is referred to as the "multilayered epithelium"15 of $\mathrm{BE}$, with columnar-lined epithelium overlying and slightly compressing the underlying squamous epithelium. Possibly MAP-infected cardiac surface mucus cells sometimes migrate underneath rather than over esophageal squamous epithelium, referred to as "subsquamous"16 or "buried" BE, which while usually considered a post-ablation artifact is actually common in untreated BE patients ${ }^{17}$.

In contrast to goblet cell hyperplasia, the proliferation of intestinal-type goblet cells in the stomach, a location where intestinal goblet cells don't normally exist, is called intestinal metaplasia or goblet cell metaplasia. The possibility that MAP can cause the proliferation of gastric or intestinal type columnar-lined epithelium is suggested by the known ability of Helicobacter pylori (HP) to cause intestinal metaplasia. Long term gastric HP infection results first in complete metaplasia, consisting of small intestinal enterocytes and small intestinal-type goblet cells, and then incomplete metaplasia, consisting of colonic-type goblet cells. These colonic-type goblet cells are the immediate precursor lesion of HP-associated gastric cancer ${ }^{18}$. After first causing the proximal proliferation of gastric cardiac mucus cells, MAP may 'do' the same thing as HP, causing their complete, and then incomplete, intestinal metaplasia.

Depending on the country, the diagnosis of BE requires the presence of goblet cells. Goblet cell-containing columnar-lined epithelium or intestinal metaplasia is a requirement for the diagnosis of $\mathrm{BE}$ in the United States and continental Europe, but in the United Kingdom and Japan only columnar-lined epithelium is required ${ }^{19}$. Columnarlined epithelium in Barrett's esophagus has four histologic subtypes, two gastric metaplasias and two intestinal metaplasias. The two types of gastric metaplasia, gastric cardia-type epithelium and gastric fundic-type epithelium, do not contain goblet cells. The two types of intestinal metaplasia, incomplete or small intestinal type, and complete or large intestinal type, do contain goblet cells ${ }^{20}$. Just as chronic HP infection results in the two histologic subtypes of intestinal metaplasia in the stomach, chronic MAP infection of the gastric cardia and esophagus may cause all of the histologic subtypes of gastric and intestinal columnar-lined epithelium in the esophagus, all of which are precursors of EAC.
Researchers are divided on the relationship between gastroesophageal reflux disease (GERD), BE and EAC. Some researchers believe that all cases of $\mathrm{BE}$ are preceded by GERD or reflux esophagitis. They explain the lack of preceding GERD in some patients with BE or EAC by arguing that not all cases of GERD are symptomatic. If GERD is a necessary precursor of $\mathrm{BE}$, then MAP must be able to invade squamous epithelium, causing the acute inflammation of reflux esophagitis. There are no studies of MAP infection of the upper gastrointestinal tract in animals, despite the common perception that MAP infection in animals occurs exclusively by the fecal-oral route. Esophageal Crohn's disease is characterized by ulcers and chronic inflammation as well as strictures ${ }^{7}$. It is possible that MAP infection of esophageal squamous epithelium may result in the acute inflammation of GERD.

Pathologists have, however, noted that the damaged squamous epithelium, including ulcers and erosion, of reflux esophagitis/GERD in humans is replaced by regenerating squamous epithelium, not by Barrett's columnar mucus epithelium $^{21}$. The majority of patients with EAC and BE do not have a clinical history of GERD ${ }^{21}$. If BE is not a direct pathologic sequela of GERD, what aspect of MAP might explain the close association between MAP and GERD?

The usually close association between GERD and BE may be explained by MAP's requirement for exogenous iron and MAP's possible preference for acidic environments. MAP can't make its own iron and therefore requires exogenous iron. The acid of GERD may 'help' MAP by making iron more soluble and therefore absorbable ${ }^{22}$, which may enhance the growth and/or persistence of MAP in the esophagus. Acidity enhances but is not required for the growth of MAP in soil ${ }^{23}$. Acidity enhances but is not required for the growth of nontuberculous mycobacteria such as MAP in natural bodies of water ${ }^{24}$. Similarly, the acidic environment of GERD may enhance MAP's ability to colonize gastric cardia surface mucus or surface mucus cells but is probably not required.

The majority of EAC cases that arise in BE don't actually arise in goblet cell-containing epithelium. Instead, cardiac mucus epithelium surrounds the majority of minute esophageal adenocarcinomas ${ }^{25}$. A recent study showed that the greater the number of goblet cells in $\mathrm{BE}$, the less the risk of $\mathrm{EAC}^{26}$. The presence of goblet cells may indicate a more 'successful' immune response to continuing MAP infection, as goblet cell hyperplasia is a protective mechanism of the intestines against other enteric pathogens ${ }^{10}$. In murine intestinal Citrobacter rodentium (CR) infection, for example, goblet cell hyperplasia occurs not during the initial phases of the infection, but only during the resolving phase when the CR organism has been successfully eliminated ${ }^{27}$. Goblet cell hyperplasia is a mechanism by which the intestines successfully expel the rodent helminth Nippostrongylis 
brasiliensis $^{28}$. The studies showing MAP causing acute and chronic goblet cell hyperplasia place MAP organisms in the underlying lamina propria ${ }^{11,12}$, suggesting MAP-induced goblet cell hyperplasia does not successfully expel the organism, but may indicate a more aggressive immune response to MAP that helps contain the organism.

Why MAP may have a predilection for gastric cardiac mucus surface cells rather than fundic or pyloric surface mucus cells is unknown, but may involve MAP's possible preference for the sulphomucins and sialomucins produced by cardiac glands.

HP infection is inversely correlated with Barrett's esophagus ${ }^{29}$, suggesting HP colonization of gastric mucus precludes MAP colonization. HP colonization of gastric mucus may crowd out MAP from doing so.

MAP's perhaps preferential colonization of the acidic cardiac mucus and/or cardiac mucous surface cells may also explain the inverse relationship between idiopathic inflammatory bowel disease and $\mathrm{BE}^{30}$. Having colonized MAP's perhaps preferred location, MAP organisms may not descend further into the intestinal tract.

The common assumption that cancers in general are a result of unspecified chronic inflammation is not supported by the pathology. GERD for example is chronically inflamed squamous epithelium, yet GERD is not associated with an increased risk of esophageal squamous cell carcinoma. Instead, species of fungi ${ }^{31}$ and human papillomavirus types 16 and $18^{32}$ are associated with esophageal squamous cell carcinoma.

The possibility that MAP may be involved in the pathogenesis of BE and associated EAC does not rule out the effect of co-factors on the development of EAC. As high salt diets and smoking 'help' HP cause gastric cancer ${ }^{33}$, co-factors including obesity and high sucrose diets may 'help' MAP cause EAC. Obesity is associated with an increased risk of infection ${ }^{34}$. Adipocytes are storage cells for Mycobacterium tuberculosis organisms ${ }^{35}$; similarly, adipocytes may be storage cells for MAP organisms, possibly explaining the relationship between obesity and $\mathrm{EAC}^{36}$. MAP is more invasive in hyperosmolar, high lactose, environments ${ }^{37}$, and may be more invasive in high sucrose western diet environments, possibly explaining the relationship between high sugar diets and an increased risk of $\mathrm{BE}^{38,39}$ and $\mathrm{EAC}^{40}$.

As an enteric pathogen, MAP organisms or antibodies to MAP organisms may be present in EAC and BE patients' esophagi, serum and feces. The regression or resolution of $\mathrm{BE}$, and an improved survival rate of EAC patients treated with anti-MAP therapies in addition to standard surgical and chemotherapy, including dietary alterations such as the specific carbohydrate diet, anti-MAP antibiotics such as RedHill Biopharma's RHB-104 triple antibiotic combination and anti-infective ultraviolet blood irradiation would lend additional support to this hypothesis.

\section{Acknowledgments}

As always, my research would not be possible without the assistance of Dr. Beth Hill at the Providence Sacred Heart Medical Center and Children's Hospital's Health Sciences Library in Spokane, Washington as well as the other libraries that participate in the FreeShare Library group within the DOCLINE National Network of Libraries of Medicine. My personal gastroenterologist, Ajay Pabby, M.D., M.P.H. heard about MAP and the goblet cell hyperplasia lesion of colorectal cancer and immediately thought of this idea.

\section{References}

1. Pohl H, Welch HG. The role of overdiagnosis and reclassification in the marked increase of esophageal adenocarcinoma incidence. J Natl Cancer Inst. 2005; 97(2): 142-6.

2. Thrift AP, Whiteman DC. The incidence of esophageal adenocarcinoma continues to rise: analysis of period and birth cohort effects on recent trends. Ann Oncol. 2012; 23(12): 3155-62.

3. Thrift AP. The epidemic of oesophageal carcinoma: Where are we now. Cancer Epidemiol. 2016; 41: 88-95.

4. Liu S, Dai JY, Yao L, et al. Esophageal Adenocarcinoma and Its Rare Association with Barrett's Esophagus in Henan, China. PLoS One. 2014; 9(10): e110348.

5. Davis WC, Kuenstner JT, Singh SV. Resolution of Crohn's (Johne's) disease with antibiotics: what are the next steps. Expert Rev Gastroenterol Hepatol. 2017; 11(5): 393-396.

6. Katsanos KH, Torres J, Roda G, et al. Review article: non-malignant oral manifestations in inflammatory bowel diseases. Aliment Pharmacol Ther. 2015; 42(1): 40-60.

7. Decker GA, Loftus EV, Jr., Pasha TM, et al. Crohn's disease of the esophagus: clinical features and outcomes. Inflamm Bowel Dis. 2001; $7(2):$ 113-9.

8. Davis KG. Crohn's Disease of the Foregut. Surg Clin North Am. 2015; 95(6): 1183-93, vi.

9. Molicotti P, Scanu AM, Lumbau A, et al. Molecular identification of Mycobacterium avium subspecies paratuberculosis in oral biopsies of Crohn's disease patients. Gut Pathog. 2013; 5(1): 18.

10.Kim JJ, Khan WI. Goblet cells and mucins: role in innate defense in enteric infections. Pathogens. 2013; 2(1): 55-70.

11. Khare S, Nunes JS, Figueiredo JF, et al. Early phase morphological lesions and transcriptional responses of bovine ileum infected with Mycobacterium avium subsp. paratuberculosis. Vet Pathol. 2009; 46(4): 717-28.

12. Charavaryamath C, Gonzalez-Cano P, Fries P, et al. Host responses to persistent Mycobacterium avium subspecies paratuberculosis infection in surgically isolated bovine ileal segments. Clin Vaccine Immunol. 2013; 20(2): 156-65.

13.Golan L, Livneh-Kol A, Gonen E, et al. Mycobacterium avium paratuberculosis invades human small-intestinal goblet cells and elicits inflammation. J Infect Dis. 2009; 199(3): 350-4.

14.Wang DH. The Esophageal Squamous Epithelial Cell-Still a Reasonable Candidate for the Barrett's Esophagus Cell of Origin? Cell Mol Gastroenterol Hepatol. 2017; 4(1): 157-160.

15. Glickman JN, Chen YY, Wang HH, et al. Phenotypic characteristics of a 
distinctive multilayered epithelium suggests that it is a precursor in the development of Barrett's esophagus. Am J Surg Path. 2001; 25(5) 569-78.

16. Mashimo H. Subsquamous intestinal metaplasia after ablation of Barrett's esophagus: frequency and importance. Current Opin Gastroenterol. 2013; 29(4): 454-9.

17. Bartel MJ, Srivastava A, Gordon S, et al. Subsquamous intestinal metaplasia is common in treatment-naive Barrett's esophagus. Gastrointest Endosc. 2018; 87(1): 67-74.

18. Semino-Mora C, Doi SQ Marty A, et al. Intracellular and interstitial expression of Helicobacter pylori virulence genes in gastric precancerous intestinal metaplasia and adenocarcinoma. J Infect Dis. 2003; 187(8): 1165-77.

19. Salimian KJ, Waters KM, Eze O, et al. Definition of Barrett Esophagus in the United States: Support for Retention of a Requirement for Goblet Cells. Am J Surg Path. 2018; 42(2): 264-268.

20. Piazuelo MB, Haque S, Delgado A, et al. Phenotypic differences between esophageal and gastric intestinal metaplasia. Mod Path. 2004; 17(1) 62-74.

21. Appelman HD, Umar A, Orlando RC, et al. Barrett's esophagus: natural history. Ann N Y Acad Sci. 2011; 1232: 292-308.

22. Michel AL, Bastianello SS. Paratuberculosis in sheep: an emerging disease in South Africa. Vet Microbiol. 2000; 77(3-4): 299-307.

23. Kopecky KE. Distribution of paratuberculosis in Wisconsin, by soil regions. J Am Vet Med Assoc. 1977; 170(3): 320-4.

24. Iivanainen EK, Martikainen PJ, Vaananen PK, et al. Environmental factors affecting the occurrence of mycobacteria in brook waters. Appl Environ Microbiol. 1993; 59(2): 398-404.

25. Takubo K, Aida J, Naomoto Y, et al. Cardiac rather than intestinal-type background in endoscopic resection specimens of minute Barrett adenocarcinoma. Hum Pathol. 2009; 40(1): 65-74.

26.Srivastava A, Golden KL, Sanchez CA, et al. High Goblet Cell Count Is Inversely Associated with Ploidy Abnormalities and Risk of Adenocarcinoma in Barrett's Esophagus. PLoS One. 2015; 10(7): e0133403.

27. Barthold SW, Coleman GL, Jacoby RO, et al. Transmissible murine colonic hyperplasia. Vet Pathol. 1978; 15(2): 223-36.

28.Turner JE, Stockinger B, Helmby H. IL-22 mediates goblet cell hyperplasia and worm expulsion in intestinal helminth infection. PLoS Pathog. 2013; 9(10): e1003698.

29. Rokkas T, Pistiolas D, Sechopoulos P, et al. Relationship between Helicobacter pylori infection and esophageal neoplasia: a metaanalysis. Clin Gastroenterol Hepatol. 2007; 5(12): 1413-7, 1417 e1-2.

30.Sonnenberg A, Turner KO, Genta RM. Decreased risk for microscopic colitis and inflammatory bowel disease among patients with reflux disease. Colorectal Dis. 2018.

31.Zhu F, Willette-Brown J, Song NY, et al. Autoreactive T Cells and Chronic Fungal Infection Drive Esophageal Carcinogenesis. Cell Host Microbe. 2017; 21(4): 478-493 e7.

32.Wang J, Zhao L, Yan H, et al. A Meta-Analysis and Systematic Review on the Association between Human Papillomavirus (Types 16 and 18) Infection and Esophageal Cancer Worldwide. PLoS One. 2016; 11(7): e0159140.

33.Wroblewski LE, Peek RM, Jr., Wilson KT. Helicobacter pylori and gastric cancer: factors that modulate disease risk. Clin Microbiol Rev. 2010; 23(4): 713-39.

34.Torres L, Martins VD, Faria AMC, et al. The intriguing relationship between obesity and infection. J Infectiology. 2018; 1(1): 6-10.

35.Neyrolles O, Hernandez-Pando R, Pietri-Rouxel F, et al. Is adipose tissue a place for Mycobacterium tuberculosis persistence. PLoS One. 2006; 1 : e43.

36. Chow WH, Blot WJ, Vaughan TL, et al. Body mass index and risk of adenocarcinomas of the esophagus and gastric cardia. J Natl Cancer Inst. 1998; 90(2): 150-5.

37.Patel D, Danelishvili L, Yamazaki Y, et al. The ability of Mycobacterium avium subsp. paratuberculosis to enter bovine epithelial cells is influenced by preexposure to a hyperosmolar environment and intracellular passage in bovine mammary epithelial cells. Infect Immun. 2006; 74(5): 2849-55.

38. Li N, Petrick JL, Steck SE, et al. Dietary sugar/starches intake and Barrett's esophagus: a pooled analysis. Eur J Epidemiol. 2017; 32(11): 1007-1017.

39. Riegler M, Kristo I, Asari R, et al. Dietary sugar and Barrett's esophagus. Eur Surg. 2017; 49(6): 279-281.

40.Li N, Petrick JL, Steck SE, et al. A pooled analysis of dietary sugar/ carbohydrate intake and esophageal and gastric cardia adenocarcinoma incidence and survival in the USA. Int J Epidemiol. 2017; 46(6): 18361846. 